\title{
Le stockage définitif des déchets radioactifs et la radioprotection*
}

\author{
H. MÉTIVIER ${ }^{1}$
}

(Manuscrit reçu le 30 septembre 2007, accepté le 27 novembre 2007)

RÉSUMÉ Pour des raisons d'efficacité opérationnelle, la CIPR a bâti un système dosimétrique s'appuyant sur l'additivité des effets sur la santé quelle que soit la nature du rayonnement et que l'exposition soit d'origine externe ou interne à l'individu exposé. Ce système a rempli son rôle, le bilan de la radioprotection est aujourd'hui jugé bon. Aujourd'hui, le challenge posé à l'énergie nucléaire est de faire la démonstration que la gestion de ses déchets éventuellement stockés en profondeur sera sans danger pour les générations futures. Le scénario envisagé est lié au retour vers la biosphère avec pour conséquence une contamination interne par ingestion de radionucléides à vie longue. Le système de protection radiologique actuel est-il adapté à ce scénario d'ingestion chronique ? Que signifie une irradiation alpha ? Que sait-on vraiment en terme dosimétriques et de risques de la chronicité des expositions ? Autant de questions pour lesquelles nous n'avons pas toujours la réponse et qu'il faut donc poser au moment où l'on préconise un dialogue avec les parties prenantes, d'autant plus que de récentes observation scientifiques remettent en cause bien des certitudes. De nouveaux programmes de recherche en radiotoxicologie sont absolument nécessaires pour répondre à ces questions légitimes, l'exemple de la démarche de l'industrie pharmaceutique pour l'obtention des autorisations de mise sur le marché des médicaments est à méditer.

ABSTRACT Final storage of radioactive waste and radiological protection.

For operational effectiveness, ICRP built a dosimetric system based on the additivity of the effects whatever are the nature of the radiation and the origin of the exposure, external or internal. This system fulfilled the assigned role; the assessment of the protection against radiation is good. Today, the challenge to overcome with regard of the nuclear energy is to make the demonstration that the management of disposed wastes in geological formations will be without risk to the future generations. The scenario considered is related to the return towards the biosphere and an internal contamination by ingestion of long-lived radionuclides. Is the current radiological protection system adapted to this situation? What means irradiation alpha? What does one really know in dosimetric and risk terms for the chronicity of internal exposures? As many questions for which we always do not have the answer and that it is thus necessary to consider at the time when one recommends a dialogue with the stakeholders and that recent scientific observations call into question many certainties. New research programmes in radiotoxicology appear absolutely necessary to answer these legitimate questions. The example of the step of pharmaceutical industry for obtaining the marketing authorizations of the drugs is to be meditated.

Keywords: long live radionuclides/waste management/deep storage/chronic exposure/radiotoxicology

* Ce papier a été présenté à l'invitation de l'Association belge de radioprotection (ABR) le 20 avril 2007 à Bruxelles. Il est publié sous une forme très proche dans les Annales de l'association belge de Radioprotection 2007, Vol. 32, N³, pp. 129-142 sous le titre : le stockage définitif des déchets radioactifs, Y a-t-il d'autres solutions pour l'opinion publique? L'auteur et le comité de rédaction de la revue Radioprotection remercient vivement Claire Stievenart, secrétaire permanente, et le bureau de l'ABR pour leur aimable autorisation de reproduire cet article dans notre journal.

12 allée des Hautes Futaies, 91450 Soisy-sur-Seine, France. 
H. MÉTIVIER

Le débat sur la gestion des déchets radioactifs à vie longue se développe actuellement dans un cadre essentiellement technique avec pour toile de fond des préoccupations de radioprotection. Quelles sont les réelles préoccupations de la société civile à cet égard, quelle est la position des radioprotectionnistes vis-à-vis du stockage de ces déchets et leur éventuel retour vers la biosphère et y a-t-il une évolution possible en la matière?

\section{Le cas de la France}

La France a engagé un effort considérable de recherche sur trois axes définis par une loi votée en 1991, communément appelé, du nom de son rapporteur «Loi Bataille ». Cette loi fixait trois axes de recherches : le tri de certains éléments radioactifs contenus dans les déchets et la réduction de leur radiotoxicité potentielle par la transmutation des radionucléides à vie longue qu'ils contiennent, la réduction de leur radiotoxicité résiduelle par l'enfouissement en profondeur, le conditionnement des déchets et la surveillance dans des installations des colis de surface (Loi, 1991).

Quinze ans après, comme le demandait la loi de 1991, le Parlement - après avoir pris connaissance des réflexions d'une Commission nationale d'évaluation indépendante (CNE, 2006) et organisé un débat public - a prolongé encore pour plusieurs années les efforts de recherches à faire, avant d'adopter une solution définitive de stockage des déchets radioactifs, désormais inscrite dans la loi.

Mais d'une loi à l'autre, y a-t-il réellement une modification des concepts envisagés pour répondre aux préoccupations réelles des populations?

\section{La notion de «parties prenantes »}

Depuis l'accident de Tchernobyl et la première loi de 1991, les dimensions sociales de la radioprotection ont considérablement changé. Tirant leçon des difficultés à gérer les conséquences de l'accident de Tchernobyl, l'agence de l'OCDE pour l'énergie nucléaire (AEN) a, par exemple, proposé une implication plus forte des parties prenantes ou «stakeholders » dans la gestion des situations de crise, mais aussi pour la gestion des déchets radioactifs (AEN, 2006).

Depuis, la Commission internationale de protection radiologique (ICRP), ainsi que d'autres agences internationales et des autorités de sûreté nationales prônent l'implication des parties prenantes dans ces situations avant la prise d'une décision. Elles en font même une condition du succès des opérations futures. Fautil encore que le dialogue s'établisse sur des bases claires répondant aux préoccupations premières des populations? 


\section{Une approche inchangée de l'estimation du risque d'apparition de cancers}

L'estimation du risque radiologique est aujourd'hui basée, excepté pour le radon, sur une approche dosimétrique, que la source d'irradiation soit externe ou interne à l'individu exposé aux rayonnements ionisants.

Pour des raisons d'efficacité opérationnelle, la CIPR a bâti un système dosimétrique et d'estimation du risque sur le concept de dose efficace, permettant l'additivité de toutes les doses d'irradiations et de leurs effets quelle que soit la nature du rayonnement et que l'exposition soit d'origine externe ou interne à l'individu exposé (ICRP, 1991). Ce système remplit parfaitement son rôle dans la gestion des situations de routine que l'on appelait «pratiques » et le bilan de la radioprotection depuis son instauration est jugé excellent.

Ce concept de dose efficace se fonde sur la notion de relation linéaire sans seuil, hypothèse non scientifiquement vérifiée, mais qui est considérée par les professionnels de la radioprotection comme un outil de gestion performant et efficace ce que rappelle la CIPR dans ses nouvelles recommandations "Use of socalled LNT is considered by the commission to be the best practical approach to managing risk from radiation exposure » (ICRP, 2007).

Cette notion a conduit toutefois à des dérives que la CIPR a voulu corriger dans ses nouvelles recommandations ; l'utilisation abusive de la dose collective, dans le temps et dans l'espace en est un exemple. La CIPR reconnaît dans son nouveau texte (ICRP, 2007) les limites de la relation linéaire sans seuil en réduisant considérablement le champ d'application de la dose collective ; «Because of this uncertainty on effects at low doses the Commission judges that it is not appropriate, for the formal purposes of public health, to calculate the hypothetical number of cases of cancer that might be associated with very small radiation doses received by large numbers of people over very long periods of time ».

\section{Les bases de la sûreté de la gestion des déchets à vie longue}

Le challenge posé à l'énergie nucléaire est de faire la démonstration que la gestion de ses déchets sera sans danger pour les générations futures.

Différentes échelles de temps sont considérées pour évaluer la sûreté des options du stockage des déchets radioactifs.

La phase opérationnelle qui est la période entre la mise en place des colis de déchets dans les installations de stockage et la fermeture du site peut prendre plusieurs décades. 
La phase dite thermique - pendant laquelle les colis de déchets génèrent une augmentation importante des températures — est estimée à 300 ans pour les déchets vitrifiés et 2000 ans pour le stockage direct du combustible usé.

La phase d'isolation durant laquelle le relargage à partir du site, de certains radionucléides dans l'argile des déchets de haute activité est négligeable, durera entre 1000 et 10000 ans après la clôture du site.

La phase géologique où le stockage entre dans les échelles géologiques de temps doit durer de 10000 ans à des millions d'années. L'impact maximal est considéré actuellement comme liée au relargage différé de radionucléides pendant cette période.

L'affirmation de non nocivité (pas de danger) est basée sur l'intégrité physique du confinement et, lorsque celui n'est plus considéré comme suffisamment sûr, sur le respect de limites de dose efficace. Il est donc nécessaire d'évaluer les doses et les risques dans des scénarios pour s'assurer du respect des objectifs fixés par les autorités de sûreté.

\section{Quel scénario de retour à l'homme?}

Le retour à l'homme se fera par la contamination des eaux de surface et de facto de la chaîne alimentaire. La contamination interne sera la conséquence d'une ingestion chronique de faibles quantités de radionucléides à vie longue, ce qui conduira à la délivrance de faibles doses à faible débit de dose.

\section{Le système actuel de radioprotection est-il adapté à cette situation exceptionnelle ?}

Le système actuel de radioprotection est basé essentiellement sur le suivi des survivants des bombardements d'Hiroshima et de Nagasaki et sur les connaissances acquises après irradiation externe. La particularité de la contamination interne est plus ou moins incorporée dans le calcul de la dose efficace par la prise en compte de facteurs de pondérations tissulaires, mais elle suppose une irradiation homogène de l'organe, ce qui n'est pas avéré, loin s'en faut avec les émetteurs alpha (Lafuma et al., 1974). De plus, nous savons que les effets du débit de dose ne sont pas négligeables bien que considérés actuellement pour les expositions externes comme variant d'un facteur 2 entre les débits de dose élevés et faibles.

Aujourd'hui, le lien entre cette approche dosimétrique bâtie pour les pratiques actuelles, la dose efficace, et le risque réel est de plus en plus contesté pour la 
contamination interne par des émetteurs à fort TEL et à vie longue (AEN, 2007). C'est exactement le scénario de la gestion du risque radiologique lié aux déchets radioactifs dans 10000 ans.

Dans son nouveau document, la CIPR accepte la remise en cause de la relation linéaire dans des situations exceptionnelles. «Although there are exceptions, for the purpose of radiological protection the Commission judges that below around $100 \mathrm{mSv}$, it is scientifically reasonable to assume that the incidence of cancer or hereditary effects will rise in direct proportion to an increase in the effective dose (dose efficace en français) ».

La CIPR reconnaît dans son nouveau texte les limites de cette relation en réduisant considérablement le champ d'application de la dose collective ; «Because of this uncertainty on effects at low doses the Commission judges that it is not appropriate, for the formal purposes of public health, to calculate the hypothetical number of cases of cancer that might be associated with very small radiation doses received by large numbers of people over very long periods of time ». C'est le cas de la gestion des déchets à long terme.

La question qui se pose dès lors est : l'ingestion chronique de faibles quantités de radionucléides à vie longue est-elle susceptible de provoquer des effets sur la santé ?

Bien que la réponse soit «non» puisque les populations du monde entier ingèrent de manière chronique de faibles quantités de radionucléides, nous n'avons pas de réponse claire prouvant que ces ingestions soient susceptibles de provoquer ou non des effets sur la santé. À ce jour, seules des études en cours en Finlande ont pour objectif de vérifier la pertinence de cette question (Salomaa et Ikäheimonen, 2005) en étudiant les effets sur la santé de l'ingestion chronique d'uranium dans l'eau de boisson.

Ces études aideront à répondre aux questions des populations locales sur l'impact sur leur santé du stockage de déchets lorsque l'éventuel retour à la surface des radionucléides à vie longue aura lieu. Cette préoccupation - si elle concerne bien évidemment les populations — s'adresse principalement à leurs descendants et les générations futures au travers de la contamination éventuelle de leur lieu de vie et de leur chaîne alimentaire par ces radionucléides.

\section{La remise en cause de cette loi linéaire pour la contamination interne}

Les récentes observations scientifiques remettent en cause bien des certitudes. Que signifie une irradiation alpha? Que sait-on vraiment en termes dosimétrique et de 
risques sur la chronicité des expositions? Autant de questions pour lesquelles nous n'avons pas toujours la réponse et qu'il faut donc continuer à se poser.

\section{La contamination chronique est-elle la somme de contaminations aiguës ?}

En termes de dosimétrie, la CIPR, dans ses publications 67 et 69 (ICRP, 1995, 1994), affirme sans vraie démonstration que la dose résultant d'une irradiation chronique est identique à celle liée à une irradiation aiguë pour une quantité de radionucléide incorporée identique :

- "The doses calculated in this report in Part 1 are for acute intakes. For chronic intakes doses may be less than those calculated here where growth is substantial during the period of intake. » (ICRP, 1994);

- "For chronic intakes, doses per unit intake could be somewhat less than those calculated here where growth is significant during the period of intake... These coefficients can also be applied to chronic intakes for protection purposes by determining the committed doses for each year's intake and summing for intakes over all years. » (ICRP, 1995).

Nous savons que l'ingestion chronique de plutonium ne suivait pas, par exemple, les schémas biocinétiques annoncés basés sur une incorporation unique (RenaudSalis et al., 1990). Des observations similaires sont rapportées pour l'uranium (Paquet et al., 2006) ainsi que pour d'autres d'autres radionucléides; ce qui a conduit récemment la CIPR à modifier son modèle dosimétrique alimentaire (ICRP, 2006).

Ces modifications ne sont pas encore intégrées dans les études dosimétriques concernant les stockages à long terme

\section{Ce que nous savons}

10000 ans après le stockage définitif, ne resteront que les radionucléides à vie longue dont les activités seront obligatoirement faibles. On peut rappeler ici par exemple les masses de radionucléides émettant le même nombre de particules alpha que l'on rapportera à $1 \mu \mathrm{g}$ de l'isotope ${ }^{210} \mathrm{Po}$ (Tab. I).

On voit bien que pour délivrer une même énergie, il faudra des quantités considérables de radionucléides à vie longue, qui peuvent d'ailleurs conduire à des impossibilités au niveau du scénario d'ingestion.

Cela amène à préciser ce qu'il faut entendre par déchets HAVL dans la perspective du long terme car il peut entretenir des confusions au niveau du public. 
TABLEAU I

Comparaison des masses de radionucléides émettant le même nombre de particules alpha. Comparison of the masses of radionuclides emitting the same number of alpha particles.

\begin{tabular}{ccc}
\hline & Période & Masse \\
\hline${ }^{210} \mathrm{Po}$ & $138,4 \mathrm{j}$ & 1 \\
${ }^{238} \mathrm{Pu}$ & 87,7 ans & 4500 \\
${ }^{226} \mathrm{Ra}$ & 1600 ans & 262000 \\
${ }^{239} \mathrm{Pu}$ & 24100 ans & 72000000 \\
${ }^{237} \mathrm{~Np}$ & 2140000 ans & 3180000000 \\
${ }^{238} \mathrm{U}$ & 4468000000 ans & 446000000000 \\
\hline
\end{tabular}

Rappelons également que l'activité spécifique de certains radionucléides limite considérablement les quantités pouvant s'accumuler dans l'organisme. Ainsi $12 \mathrm{mg}$ de ${ }^{129} \mathrm{I}$, quantité pondérable que renferme une thyroïde humaine correspond à $60000 \mathrm{~Bq}$ en unité de radioactivité. Cette radioactivité serait donc la valeur maximale que pourrait contenir la thyroïde si tout l'iode de la planète était composé du seul isotope 129.

La question est dès lors: après contamination interne chronique de radionucléides à vie longue, les schémas dosimétriques actuels sont-ils toujours valables pour ce scénario ? Seule l'expérimentation nous permettra de répondre à cette question.

Au niveau du risque, nous savons que les émetteurs alpha peuvent conduire à des réponses différentes de la relation linéaire sans seuil (Raabe et al., 1983, 1981 ; Park, 1992, 1990 ; Sanders et al., 1993, 1991 ; Hohryakov et Romanov, 1994).

L'observation des ostéosarcomes chez les peintres de cadran lumineux montre que la relation dose-effet ne répond pas à une relation linéaire sans seuil comme le montre la figure 1 (Evans et al., 1974 ; Rowland et al., 1978 ; Rowland, 1995) alors que les carcinomes des sinus de la face et des maxillaires sont plus proches d'une représentation linéaire. Ainsi la relation dose-effet varie avec la dose contrairement aux prédictions du modèle de la CIPR.

Après inhalation d'oxyde de plutonium chez le rat, Sanders montre clairement que là encore l'apparition de cancers pulmonaires ne répond pas à une relation linéaire sans seuil (Fig. 2).

Une réponse identique est observée par Park chez le chien après inhalation de ${ }^{239} \mathrm{Pu}$ mais aussi de ${ }^{238} \mathrm{Pu}$. Et si l'on se réfère à la publication de Hohryakov et Romanov (1994) concernant les travailleurs de Mayak, l'apparition de cancers, 
H. MÉTIVIER

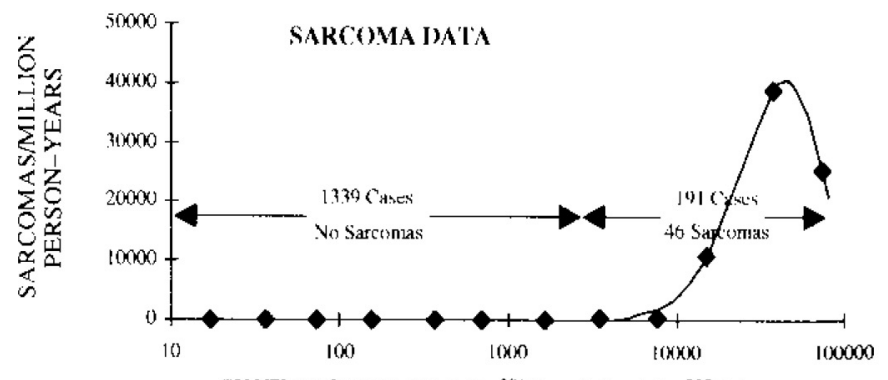

SYSTHMIC IN'TAKE (kBq $\left.{ }^{2} 20 \mathrm{Ra}+2.5 \times \mathrm{klBq}^{228} \mathrm{Ra}\right)$

Figure 1 - Relation dose-effet pour les ostéosarcomes chez les femmes peintres de cadrans lumineux au radium (d'après Rowland, 1995).

Dose effect relationship for osteosarcomas observed in women radium luminous dial painters (from Rowland, 1995).

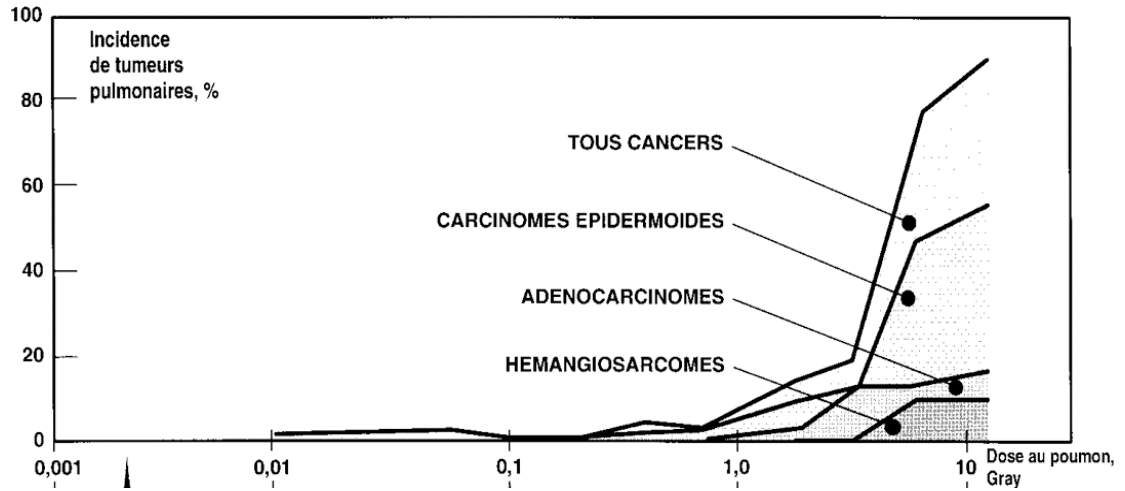

Figure 2 - Incidences de cancers pulmonaires chez le rat après inhalation d'oxyde de plutonium-239 (d'après Sanders et al., 1993, 1991).

Incidences of lung cancers in rats after inhalation of plutonium-239 oxide (from Sanders et al., 1993, 1991).

tout comme dans l'expérience de Sanders chez le rat, ne répond pas à une relation linéaire. Chez le singe, un déficit de cancers est observé après inhalation d'oxyde de ${ }^{239} \mathrm{Pu}$ si l'on se base sur une prédiction dosimétrique (Métivier et al., 1989).

À dose égale, nous savons également, comme le montre le tableau II, que plus l'irradiation des poumons par des émetteurs alpha est homogène, plus le risque est élevé (Lafuma et al., 1974 ; Dagle et al., 1988). Cette observation démentant de manière catégorique la théorie du point chaud évoquée il y a plusieurs années et qui refait malheureusement surface au gré des phantasmes des personnes peu au fait. 
TABLEAU II

Comparaison de l'incidence des cancers du poumon en fonction de l'homogénéité de l'irradiation pour un nombre de particules alpha émises identique (d'après Lafuma et al., 1974).

Comparison of lung cancers incidence according to the homogeneity of the irradiation for an identical number of alpha particles emitted (according to Lafuma et al., 1974).

\begin{tabular}{cc}
\hline & Risque cancer \\
\hline${ }^{244} \mathrm{Cm}$ nitrate & 1 \\
${ }^{238} \mathrm{Pu}$ oxyde & 0,7 \\
${ }^{238} \mathrm{Pu}$ nitrate & 0,5 \\
${ }^{241} \mathrm{Am}$ nitrate & 0,4 \\
${ }^{241} \mathrm{Am}$ oxyde & 0,3 \\
${ }^{239} \mathrm{Pu}$ nitrate & 0,2 \\
${ }^{239} \mathrm{Pu}$ oxyde & 0,2 \\
\hline
\end{tabular}

Enfin, on a remarqué, lors d'expériences de lavage pulmonaire chez le babouin, que la dose évitée par l'élimination d'oxyde de plutonium n'était pas corrélée à l'espérance de vie gagnée par l'élimination du plutonium, mais bien supérieure au seul bénéfice de la dose évitée (Nolibé et al., 1989). Ceci a été expliqué par une redistribution des particules de plutonium restant au sein du poumon puis encapsulation de l'oxyde par une coque de collagène, protégeant ainsi les cellules à risque d'une irradiation supplémentaire.

\section{Quelles études envisager alors pour répondre aux préoccupations entourant la gestion des déchets radioactifs à vie longue?}

Les observations passées sur la cancérogenèse liée à l'incorporation de radionucléides à vie longue et plus particulièrement aux émetteurs alpha montrent que la réponse dosimétrique ne permet pas d'affirmer clairement ce que seront les effets sur la santé du retour à l'homme des radionucléides à vie longue. Peu d'études sont faites par la voie de l'ingestion. De plus, nous n'avons aucune information pour les produits de fission, ${ }^{99} \mathrm{Tc},{ }^{135} \mathrm{Cs}$ et ${ }^{129} \mathrm{I}$.

Il semble donc que de nouveaux programmes de recherche soient absolument nécessaires pour répondre aux questions légitimes des populations concernées par une situation exceptionnelle. Ces programmes devraient se baser sur des études toxicologiques, aujourd'hui abandonnées, et prendre modèle sur l'industrie pharmaceutique lorsque cette dernière demande l'autorisation de mise sur le marché d'un médicament (les AMM).

Cette démarche est contraignante et bien encadrée par la réglementation européenne (CE, 2001) selon des normes qualitatives strictes de bonnes pratiques 
H. MÉTIVIER

de laboratoire. Elle demande en premier de la part des demandeurs une étude de pharmacocinétique et de toxicologie pour chaque forme galénique et pour chaque voie d'administration. Ces études animales permettent d'évaluer et de signaler les risques de toxicité qui peuvent apparaître lors des études cliniques qui seront démarrées en fonction des résultats de ces études précliniques. L'analyse de ces études animales fait partie intégrante de l'évaluation du bénéfice-risque d'une dose quotidienne et d'une durée pour un traitement d'une nouvelle molécule. Cette évaluation proposée par le demandeur d'AMM est ensuite validée ou non par les autorités de tutelle qui délivre l'AMM.

La législation précise encore qu'aucune étude in vitro même si elle peut aider à comprendre les mécanismes d'action ne peut remplacer l'étude dans un organisme entier de la toxicité du produit avec une bonne fiabilité.

Transposée au problème de gestion des déchets, l'application d'une démarche similaire devrait conduire à étudier la toxicité des radionucléides par la voie ingestion pour des doses correspondantes à celles qui sont sensées atteindre l'homme. Il faudrait donc lancer des études de toxicologie par ingestion chronique de faibles quantités des radionucléides à vie longue tels les ${ }^{135} \mathrm{Cs},{ }^{129} \mathrm{I}$ et actinides.

De premiers résultats observés en Finlande chez l'homme exposé quotidiennement à une contamination de son eau de boisson par de l'uranium naturel montre que les modèles prescriptifs de la CIPR, utilisés actuellement pour les études d'impact des déchets sont battus en brèche (Kurttio et al., 2005) et que même à des concentrations relativement élevées en uranium, aucune toxicité rénale n'est observée (Kurttio et al., 2006). Chez le rat exposé à une ingestion chronique d'uranium, il est fait état d'une accumulation et d'une distribution de l'uranium différente de celle des modèles actuels (Paquet et al., 2006). L'approche dosimétrique aujourd'hui proposée nous paraît donc dès lors devoir être complétée pour répondre aux questions du public.

\section{Conclusion}

Les recherches menées tant en France qu'en Europe pour améliorer les connaissances scientifiques sur la gestion des déchets et la recherche de solutions techniques nouvelles ont largement fait progresser nos connaissances sur ce sujet. Elles permettent d'asseoir la gestion des déchets radioactifs sur des bases scientifiques solides (CNE, 2006). En France, la loi de 1991 a aussi permis d'apporter au public une information réservée jusque-là aux experts.

Il n'en demeure pas moins que l'acceptabilité des populations n'a pas, quinze ans après l'adoption de cette loi, beaucoup progressé. Tout comme il y a quinze 
ans, on ne cherche pas, semble-t-il, à vouloir répondre de manière directe à la question posée par les populations : quel est le risque pour ma santé et celle de nos descendants si mon eau de boisson ou mes aliments sont contaminés ? Les études de radiobiologie si utiles qu'elles soient ne permettent pas aujourd'hui de répondre clairement à cette question.

Il paraît alors qu'à l'époque où tout le monde s'accorde pour engager un dialogue avec les « parties prenantes » qu'il faille élargir le champ de recherches proposées et lancer de nouvelles recherches de toxicologie pour répondre efficacement aux craintes des populations sur leur santé. La démarche efficace mise en place par l'industrie pharmaceutique pourrait et devrait servir de modèle. Cette démarche, conjuguée aux efforts à venir pour diminuer le terme source dans les déchets des réacteurs de génération IV (CEA, 2005) pourrait, modifier l'attitude du public face à ce problème récurrent de la gestion des déchets radioactifs.

Cet effort demandé doit rassembler toutes les compétences mondiales en la manière. Il pourrait tout naturellement trouver sa place dans de nouveaux programmes de recherche pour préparer les futurs réacteurs. À cet égard d'autres recherches de même type en radiotoxicologie ont été récemment proposées (Métivier et Guillaumont, 2007).

Remerciements. L'auteur tient à remercier $R$. Guillaumont (Académie des Sciences) et $S$. André (Laboratoire Roche, Bâle) pour leur aide précieuse lors de la finalisation de cet article.

\section{RÉFÉRENCES}

AEN (2006) Agence pour l'énergie nucléaire, Les attentes de la société dans la gestion des déchets radioactifs et s'y adapter, Enseignements principaux et expériences du Forum sur la confiance des parties prenantes. OCDE/AEN, Paris.

AEN (2007) Agence pour l'énergie nucléaire, Scientific issues and emerging challenges for radiation protection, Report of the Expert group on the implications of radiation protection science. OCDE/AEN, Paris.

CE (2001) Commission européenne, directive 2001/83/CE du parlement européen et du conseil du 6 novembre 2001 instituant un code communautaire relatif aux médicaments à usage humain, Journal officiel des communautés européennes du 28 novembre 2001, L311/74-L311/76.

CEA (2005) Commissariat à l'énergie atomique, L'énergie nucléaire du future: quelles recherches pour quels objectifs, Une monographie de la Direction de l'énergie nucléaire. CEA Saclay et Groupe Moniteur, Paris.

CNE (2006) Commission nationale d'évaluation des recherches sur la gestion des déchets radioactifs, Rapport Global d'évaluation, janvier 2006.

Dagle G.E., Park J.F., Gilbert E.S., Weller R.E. (1988) Risk estimates for lung tumours from inhaled ${ }^{239} \mathrm{PuO}_{2},{ }^{238} \mathrm{PuO}_{2},{ }^{239} \mathrm{Pu}\left(\mathrm{NO}_{3}\right)_{4}$ in beagle dogs, Radiat. Prot. Dosim. 26, 61-72.

Evans R.D., Keane A.T., Shanahan M.M. (1974) Radium in man, Health Phys. 27, 497-519.

Hohryakov V.F., Romanov S.A. (1994) Lung cancer in radiochemical industry workers, Sci. Total Environn. 142, 25-28.

ICRP Publication 60 (1991) 1990 Recommendations of the International Commission on radiological Protection, Ann. ICRP 21 (1-3). 
ICRP publication 67 (1994) Age-dependent doses to members of the public from intake of radionuclides: Part 2, Ingestion dose coefficients, Ann. ICRP 23 (3/4).

ICRP publication 69 (1995) Age-dependent doses to members of the public from intake of radionuclides: Part 3, Ingestion dose coefficients, Ann. ICRP 25 (1).

ICRP publication 100 (2006) Human alimentary tract model for radiological protection, Ann. ICRP 36 (1-2).

ICRP publication 103 (2007) Recommendations of the ICRP, Ann. ICRP 37 (2-3).

Kurttio P., Komulainen H., Leino A., Salonen L., Auvinen A., Saha H., Komulainen H. (2005) Bone as a possible target of chemical toxicity of natural uranium in drinking water, Environm. Health Persp. 113, 68-72.

Kurttio P., Harmoinen A., Saha H., Salonen L., karpas Z., Komulainen H., Auvinen A. (2006) Kidney toxicity of ingested uranium from drinkung water, Am. J. Kidney Dis. 47, 972-982.

Lafuma J., Nénot J.C., Morin M., Masse R., Métivier H., Nolibé D., Skupinski W. (1974) Respiratory carcinogenesis in rats after inhalation of radioactive aerosols of actinides and lanthanides in various chemical forms, In Experimental lung cancer, E. Karbe, J.F. Park (Eds). Springer Verlag, New York, Vol. 1, pp. 443-453.

Loi (1991) loi n ${ }^{\circ} 91-1381$ du 30 décembre 1991 relative aux recherches sur la gestion des déchets radioactifs, JORF n ${ }^{\circ} 1 \mathrm{du}^{\mathrm{er}}$ janvier 1992.

Métivier H., Guillaumont R. (2007) Mieux connaître le devenir biologique des futurs matériaux nucléaires, une nécessité pour maintenir un bon niveau de radioprotection, Radioprotection $\mathbf{4 2}$, 315-335.

Métivier H., Masse R., Rateau G., Nolibé D., Lafuma J. (1989) New data on the toxicity and translocation of inhaled ${ }^{239} \mathrm{PuO}_{2}$ in baboons, Radiat. Prot. Dosim. 26, 167-172.

Nolibé D., Métivier H., Masse R., Chrétien J. (1989), Benefits and risks of bronchopulmonary lavage: a review, Radiat. Prot. Dosim. 26, 337-343.

Paquet F., Houpert P., Blanchardon E., Delissen O, Maubert C., Dhieux B., Moreels A.M., Frelon S., Gourmelon P. (2006) Accumulation and distribution of uranium in rats after chronic exposure by ingestion, Health Phys. 90, 139-147.

Park J.F. (1990) Inhaled plutonium oxide in dog, In Pacific Northwest laboratory Report for 1989 to the DOE Office of Energy Research Part I: Biomedical Sciences. Springfield, VA. National Technical Information Service, pp. 11-28, 101-107.

Park J.F. (1992) Inhaled plutonium oxide in dog, In Pacific Northwest laboratory Report for 1991 to the DOE Office of Energy Research Part I: Biomedical Sciences. Springfield, VA. National Technical Information Service.

Raabe O.G., Park N.J., Book S.A. (1981) Dose-response relationship for bone tumors in beagle exposed to ${ }^{226} \mathrm{Ra}$ and ${ }^{90} \mathrm{Sr}$, Health Phys. 40, 863-880.

Raabe O.G., Book S.A., Parks N.J. (1983) Lifetime bone cancer response relationships in beagles and people from skeletal burdens of ${ }^{226} \mathrm{Ra}$ and ${ }^{90} \mathrm{Sr}$, Health Phys. 44 (Suppl 1), 33-48.

Renaud-Salis V., Lataillade G., Métivier H. (1990) Effect of mass, oxidation state and duration of chronic ingestion on plutonium absorption in fed rats, Int. J. Radiat. Biol. 58, 691-704.

Rowland R.E., Stehey A.F., Lucas H.F (1978) Dose response relationships for female radium dial workers, Radiat. Res. 76, 368-383.

Rowland R.E. (1995) Dose-response relationships for female radium dial workers: a new look, In Health effects of internally deposited radionuclides: Emphasis on Radium and Thorium, G. van Kaick, A. Karaoglou, A.M. Kellerer (Eds). World Scientific, Singapore, pp. 135-143.

Salomaa S., Ikäheimonen T.K. (2005) Research activities of STUK 2000-3004, STUK report A210, Helsinki.

Sanders C.L., Lauhala K.E., Mahaffey J.A. (1991) Low-level ${ }^{239} \mathrm{PuO}_{2}$ Life-span Studies, In Pacific Northwest laboratory Report for 1990 to the DOE Office of Energy Research Part I: Biomedical Sciences. Springfield, VA. National Technical Information Service, pp. 39-44.

Sanders C.L., Lauhala K.E., McDonald K.E. (1993) Lifespan studies in rats exposed to aerosol. III. Survival and lung tumours, Int. J. Radiat. Biol. 64, 417-430. 